\title{
Exploring isospin effects on the level density parameter
}

\author{
P. Marini ${ }^{1,2,3, a}$, M.F. Rivet ${ }^{2}$, B. Borderie ${ }^{2}$, N. Le Neindre ${ }^{4}$, A. Chbihi $^{1}$, G. Verde ${ }^{5}$, and J.P. Wieleczko ${ }^{1}$ et al. \\ for the INDRA-VAMOS collaboration \\ 1 GANIL, CEA/DSM-CNRS/IN2P3, B.P.5027, F-14050 Caen cedex, France \\ 2 Institut de Physique Nucléaire, IN2P3-CNRS, F-91406 Orsay cedex, France \\ 3 Dipartimento di Fisica dell'Università and INFN, Bologna, Italy \\ 4 LPC, CNRS/IN2P3, ENSICAEN, Université de Caen, F-14050 Caen cedex, France \\ 5 INFN-Sez. CT and Università di Catania, I-95100 Catania, Italy
}

\begin{abstract}
Ar+Ni reactions have been studied to fix some constraints on the isospin dependence of the level density. The coupling of INDRA and VAMOS apparatuses allows to obtain highly exclusive data . The preliminary results on the $\mathrm{Ar}+\mathrm{Ni}$ fusion-evaporation cross sections suggest a dependence on the entrance channel mass asymmetry.
\end{abstract}

\section{Introduction}

One of the main goals of the current research in nuclear physics in the low and intermediate energy domain is to improve our knowledge on the competition between the occurring reaction mechanisms and the dependence on the characteristics of the entrance channel.

The advent of radioactive beams, as the ones produced by the SPIRAL facility, coupled to judiciously chosen targets, allows for the very first time to explore the properties of a large number of isotopes of compound nuclei of a given $Z$ and, consequently, to test the influence of the mass asymmetry of the entrance channel on the fusion cross section. Fusion reactions, where the composite system deexcites mainly by evaporation, allow to experimentally access the nuclear level density $\rho(E)$ and the limiting temperature $T_{\text {lim }}$, in order to study their $N / Z$ dependence.

The nuclear level density is an important quantity for the study of both thermal and decay properties of excited nuclei and for the determination of cross sections used in nuclear astrophysics calculations $[1,2]$. Moreover $\rho$ is an essential ingredient in calculating the statistical decay of a compound nucleus $(\mathrm{CN})$ in statistical models. The knowledge of the level density is thus highly needed in all regimes of excitation energies and for the full range of $\mathrm{Z}$ and $\mathrm{N}$, from the $\beta$ line of stability to the drip lines.

At present empirical parametrizations extrapolating the level density parameter $a$ towards the p-drip line are available [4], see Fig.1. However within the $N / Z$ range produced by stable beams, contradictory results have been found [3-5]. It is therefore of primary importance to test the effect of the isospin on level densities through the evaporative charged particle emission by forming more exotic systems such as those produced by the radioactive beams available today and in future facilities.

a e-mail: marini@ganil. fr
The aim of the experiment is to explore the variation of deexcitation properties and thus level density parameters with the $N / Z$ of the Pd compound nucleus when going from stable nuclei to the proton drip line. It will allow to fix some constraints on the $N / Z$ dependence of $a$ (see Fig.1). Within this framework the aim of this preliminary study is to have quantitative information on the $N / Z$ dependence of the $\mathrm{Ar}+\mathrm{Ni}$ fusion cross section.

\section{Experiment}

${ }^{34} \mathrm{Ar},{ }^{36} \mathrm{Ar}$ and ${ }^{40} \mathrm{Ar}$ ion beams with energies of $\sim 13 \mathrm{AMeV}$ have been accelerated and impinged onto $300 \mu \mathrm{g} / \mathrm{cm}^{2}$ thick, self-supporting, isotopically enriched Ni targets $\left({ }^{58} \mathrm{Ni},{ }^{60} \mathrm{Ni}\right.$ and ${ }^{64} \mathrm{Ni}$ ) in order to produce fused $\mathrm{Pd}$ nuclei, with mass number varying from 92 to 104 . The $N / Z$ of the compound systems ranges from $1.00\left({ }^{92} \mathrm{Pd}\right)$ to $1.26\left({ }^{104} \mathrm{Pd}\right)$.

The thermodynamical properties of different isotopes produced in quite the same conditions of formation and detection are studied precisely choosing the incident energies for each projectile to get the same excitation energy per nucleon of compound nuclei $(\sim 2.9 \mathrm{AMeV})$.

$\sim 13 \mathrm{AMeV}$ beam energy is a compromise to reduce the preequilibrium effects and have sufficient recoil energy for nuclear charge identification of residues. At this incident energy complete and incomplete fusion reaction mechanisms are both present. Recoil energy criteria should allow to determine whether the observed reactions are mostly complete or incomplete fusion ones $[7,8]$.

The experiment has been performed taking advantage of the coupling of the $4 \pi$ detector INDRA $[9,10]$ and the VAMOS spectrometer [11].

The INDRA multidetector, designed to study the deexcitation properties of hot nuclei produced during a heavy ions collision, has a structure in rings centered on the beam axis. Telescope detectors, constituted by an ionization chamber,

This is an Open Access article distributed under the terms of the Creative Commons Attribution-Noncommercial License, which permits unrestricted use, distribution, and reproduction in any noncommercial medium, provided the original work is properly cited. 


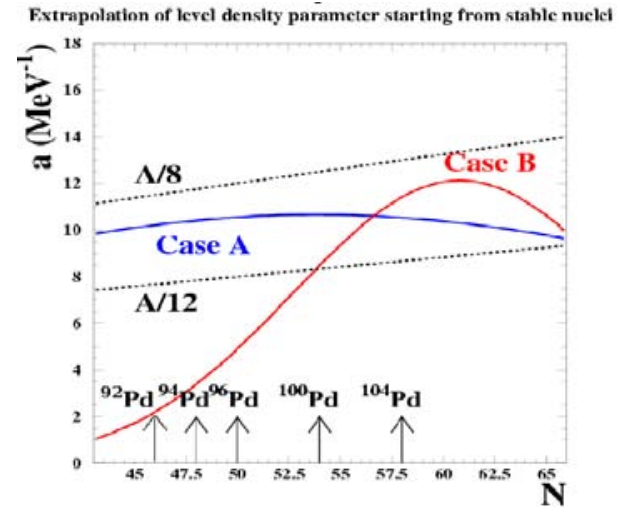

Fig. 1. Evolution of the level density parameter according to two different parametrisations for different Pd isotopes (see Ref.[4]). Experimental values $A / 8$ and $A / 12$ obtained for low and high excitation energies respectively [6] are also reported.

a high resolution silicon detector and a cesium iodide scintillator, provide the detection and the identification of all reaction products.

The large acceptance mass spectrometer VAMOS has been designed to select and identify $(Z, A$ and $E$ ) heavy reactionproducts of interest. The detection system at the focal plane of VAMOS consists of two emissive foils coupled with an ionization chamber and a silicon detector wall, which provide $\Delta E, E, Z$, position and time-of-flight measurements. The scattering angle at the target $(\theta), B \rho$ parameters and the mass $A$ of each particle are obtained by software trajectory reconstruction.

The coupling of these two detectors allows to have an event by event complete information on the evaporation residue and the associated light charged particles (LCP), and at last, but not the least for importance, on the neutron multiplicity by means of mass conservation. All decay chains can be measured, obtaining the percentage with which different chains lead to the same residue. The correct weighting of the different exit channels, which was never measured up to now, allows to put constraints on the values of $a$ for nuclei along the deexcitation chain. Moreover INDRA, able to measure variation on the slope of the kinetic energy spectra for all reaction products, provides information on temperature for all decay chains.

Further details on the experimental set-up can be found in Ref. [12].

\section{Analysis}

The essential information for the measurement of the differential cross section for the $\mathrm{Ar}+\mathrm{Ni}$ fusion-evaporation (FE) reaction consists in the determination of the amount of the total collected FE events at each polar angular range. In this preliminary phase only INDRA data have been analyzed. FE residues must be discriminated from fragments produced in other reaction mechanisms. The transition between the three classes of phenomena (Fusion-Evaporation (FE) and Fusion-Fission (FF) reactions, Deep Inelastic Collision and Quasi Elastic processes) occurring in heavy ion reactions at low energy is smooth. As QE processes and $\mathrm{CN}$ formations correspond to very different impact parameters, the reaction products kinetic energies are very different from each other, allowing an easy separation, in $\Delta E-$ $E$ spectra, of $\mathrm{FE}$ (residue region) from quasi-elastic products. This is not true for DIC and fusion processes, where the involved $b$ are only slightly different or even equal.

Being performed up to now neither energy and time of flight calibrations, nor $Z$ and $A$ identifications, the fragment selection that can be performed is inclusive and based on general considerations and thus preliminary. Later on more exclusive fragment selection could be done, by multidimensional analysis methods.

FE reactions are characterized by the presence, in the exit channel, of only one heavy fragment (residue) with mass close to the total system mass, and a certain number of light particles. GEMINI calculations predict a FE residue charge distribution spanning from 30 to 40 for the analysed reactions.

DIC are, instead, binary process, which nearly preserves the identity of the colliding ions: quasi-projectile, QP, and quasi-target, QT, spread over the whole available $Z$ and $A$ ranges, but are centered in the vicinity of the charges and masses of the projectile and the target.

In $280 \mathrm{MeV}{ }^{40} \mathrm{Ar}+{ }^{58} \mathrm{Ni}$ measurement [13] it is shown that the most significant contribution to the DI cross section is given by fragments with $Z$ within \pm 6 unit from the initial value, i.e.

$$
Z_{Q P}=12 \div 24 \text { and } Z_{Q T}=22 \div 34
$$

We remark that the QT and the FE residue charge distributions overlap, avoiding a residue identification based on the fragment charge.

The next step is to verify if QT nuclei produced in DIC lie in the residue region, and, eventually, to evaluate their contribution to the statistics in the residue region. To this aim a code (DIFFIL) computing the kinematics for elastic diffusion, fission and deep inelastic fully relaxed reactions have been used, combined with GEMINI simulation.

The simulation, once filtered by the experimental filter, can be useful to have an idea of the residue region position in $\Delta E-E$ spectra for different values of the residue emission angle $\theta$.

For each ring, the computation of the energy lost in the ionization chamber and silicon detectors by QT nuclei, emitted at the maximum and minimum angles covered by the detector, have been made by DIFFIL code. The calculations have been performed for both $Z_{Q T}=28$ and $Z_{Q T}=$ 34. It individuates, on a $\Delta E-E$ simulated matrix, the extension of the QT region. Plotting these spectra for all the INDRA rings, we observe that increasing the angle, the deep inelastic contribution increases. QTs populate both the low energy region below the Bragg peak, where the residue number increases increasing the observed angle, and the region related to a high energy loss in silicon detector, up to the elastic region (which is not reported in the picture). Increasing the observed angle, the overlap between the residue region and the deep inelastic region increases. Therefore, to evaluate the total number of residues 


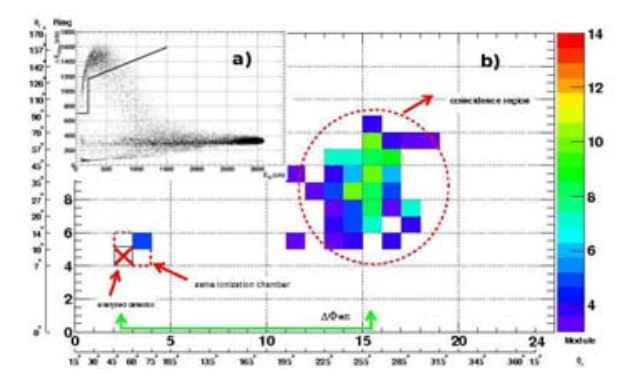

Fig. 2. a) Selection of a residue region on a $\Delta E_{C h I o}-E_{S i}$ spectrum in ${ }^{40} \mathrm{Ar}+{ }^{60} \mathrm{Ni}$ reaction. The selected residue region includes the Bragg peak. b) Ring vs. module plane.

in each ring, it becomes more and more important to take into account the DIC contribution.

The code developed to this aim allows to select a region in a $\Delta E-E$ spectrum (see Fig.2a) and to draw a bidimensional plot, which shows, in ring vs module plane (Fig.2b), the number of telescope hit by a heavy fragment $(Z \geq 12)$ in coincidence with the one lying in the residue region (coincidence region). The analysis of heavy fragments multiplicity and their angular correlations suggests the kind of the occurred reaction mechanism.

Most of the counts are confined in a restricted region where the reaction plane is located ( $\Delta \Phi=\pi$, see Fig.2b), suggesting the presence, in the selected region, of DI fragments.

We observe that, expanding the selected residues region the corresponding coincidence region in ring vs module plane expands, being centered around the same telescope. This behaviour is consistent with the fact that deep inelastic region lies between the residues and the elastic ones, and, expanding the selected region, a part of deep inelastic region is included.

Moreover the DI contribution to the residues region increases as the observed polar angle increases. As predicted by the previous analysis, the DI contribution is more and more significant for less forward rings. In ring 7 a large percentage of deep inelastic contribution lies in the region below the Bragg peak. In this case it is impossible to separate deep inelastic from fusion-evaporation events, so that the obtained total residues number will set just the upper limit. The chosen criterion to select the residue region is that the contribution to the residual count of the deep inelastic products must be limited to $\sim 1 \%$. In backward rings the tolerated DI contribution is greater (up to $\sim 20 \%$ in ring 7 for the ${ }^{40} \mathrm{Ar}+{ }^{60} \mathrm{Ni}$ reaction), being the two regions more overlapped.

Once selected the residue region in each detector, following these criteria, the number of fragments lying in this region has been counted.

Thanks to the axial symmetry of the fusion evaporation reactions, the number of evaporation residues has been evaluated in a selected $\Delta \phi$ region for each ring, in order to exclude the detectors not properly working. The accurate beam alignment has been verified and the eventual misalignment taken into account.

For each ring the total number of detected residues in a detector is the mean value over the measured values in the

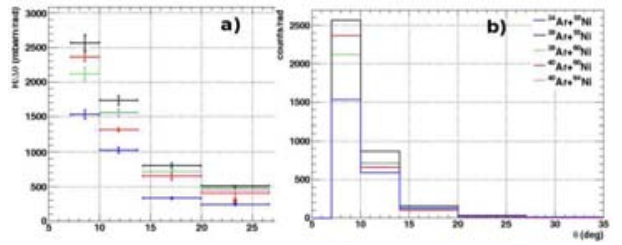

Fig. 3. Measured and GEMINI simulated differential fusionevaporation cross sections. When only 4 points are visible, the $5^{\text {th }}$ is superimposed.

$n$ modules of the $\Delta \phi$ selected region, and its uncertainty takes into account the systematic uncertainty introduced by the DI contribution present in the residues region.

The ER cross section has been normalized with respect to the yields of elastic scattered beam particles measured in first INDRA ring $\left(\Delta \theta=7^{\circ} \div 10^{\circ}\right)$. To have the complete elastic scattering angular distributions a theoretical calculation [14], based on a purely microscopic model[15], has been performed for all the reactions. The optical potential is deduced by a double folding potential.

\section{Experimental results}

Differential fusion-evaporation cross sections measured for $\mathrm{Ar}+\mathrm{Ni}$ and results obtained with GEMINI simulation, normalized to experimental data, are displayed in Fig.3 for comparison. Both distributions show the same trend: a strong decrease of $d \sigma_{F E} / d \theta$ as the CN mass decreases. These results are preliminary since the measured angular distributions are not complete and the maxima of the distributions are missed. A further analysis including the VAMOS data will allow to get information also on the peak region of the angular distributions.

Experimental distributions are broader than GEMINI ones, but the ratios of counts in the first two rings $\left(\bar{\theta}=8.5^{\circ}\right.$ and $12^{\circ}$ ) have the same trend, giving confidence in a proper description, by GEMINI, of the occurring physics processes. Based on GEMINI it is then possible to extrapolate the FE cross sections and compare them with the values reported in literature. In Tab. 1 the percentage of measured $\sigma_{F E}$ is reported, showing an increase from the $n$-rich to the $n$-poor systems. The extrapolation of $\sigma_{F E}$ is, of course, very sensitive to possible uncertainties, being the measured angular distributions less than $25 \%$ of the total angular distributions. In order to be independent from GEMINI calcula-

\begin{tabular}{c|ccc|c}
\hline & $\% \sigma_{F E}^{\text {tot }}$ & $\sigma_{F E}$ ext & $\sigma_{\text {reac }}$ & $\sigma_{F E}^{I I}$ \\
\hline${ }^{92} \mathrm{Pd}$ & $26.037 \%$ & $780 \pm 39$ & 2497 & $317 \pm 64$ \\
${ }^{94} \mathrm{Pd}$ & $23.599 \%$ & $1598 \pm 72$ & 2562 & $532 \pm 125$ \\
${ }^{96} \mathrm{Pd}$ & $23.455 \%$ & $1417 \pm 57$ & 2606 & $453 \pm 86$ \\
${ }^{100} \mathrm{Pd}$ & $18.675 \%$ & $1680 \pm 134$ & 2713 & $434 \pm 95$ \\
${ }^{104} \mathrm{Pd}$ & $17.626 \%$ & $1953 \pm 210$ & 2801 & $499 \pm 178$
\end{tabular}

Table 1. Percentage of measured angular distribution obtained by a GEMINI comparison ( $\left.\% \sigma_{F E}^{t o t}\right)$, extrapolated $\sigma_{F E}$ and reaction cross section [16]. The FE cross sections are expressed in mbarn 


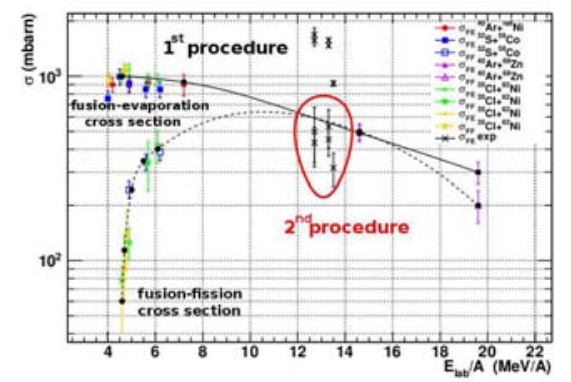

Fig. 4. Experimental results found in literature for both fusionevaporation (full symbols) and fusion-fission (open symbols) cross sections. The lines are only to drive the eyes.

tion, a new procedure is actually under analysis. Taking advantage of the expected gaussian form of the differential FE cross section $(d \sigma / d \Omega)$, a linear fit of $\ln (d \sigma / d \Omega)$ vs. $\sin ^{2} \theta$ allows to determine the analytic shape of $d \sigma / d \theta$. The integral of such function gives the $\sigma_{F E}$. Work are still in progress on these data and preliminary values are reported in Tab.1.

Experimental results, both for FE and FF reactions, found in literature are displayed in Fig.4. Data are for ${ }^{40} \mathrm{Ar}+{ }^{n a t} \mathrm{Ni}$ $[17,18],{ }^{32} \mathrm{~S}+{ }^{59} \mathrm{Co}[19],{ }^{40} \mathrm{Ar}+{ }^{68} \mathrm{Zn}[20]$ and ${ }^{35} \mathrm{Cl}+{ }^{62} \mathrm{Ni}[21$, 22]. On these basis a FE cross section of about 600 mbarn is expected for ${ }^{40} \mathrm{Ar}+{ }^{60} \mathrm{Ni}$. We would like to remark that the FF cross section is comparable with the FE one, but FF events have been removed during the analysis. The high cross section values obtained with the first procedure are not in agreement with the values present in literature, while the others show a better agreement. The main uncertainty in the first procedure comes from the assumption of identical shapes of the angular distributions in the experiment and in GEMINI. For the two procedures the absolute values rely on the parametrization of $\sigma_{e l} / \sigma_{\text {Ruth }}$.

However, independantly of the extrapolation procedure, a strong decrease of the fusion-evaporation cross section is observed for the lighter system, ${ }^{92} \mathrm{Pd}$. Being the decrease of $\sigma_{F E}$ bigger than the $\sigma_{\text {reac }}$ one, it cannot be explained in terms of a smaller number of partial waves participating to the reaction. Moreover the decrease of $\sigma_{F E}$ can be due neither to the behaviour of $\sigma_{\text {Ruth }}$, which has the opposite trend, nor to the percentage of measured $d \sigma / d \theta$, which increases when decreasing the $\mathrm{CN}$ mass. We verified that the effect can neither be ascribed to a detector threshold effects, which is less than $0.3 \%$ for all the analyzed reactions. All these observations are summarized in Fig.5. The decrease of $\sigma_{F E}$ suggests then a possible dependence of the fusion-evaporation cross section from the isospin of the reactions.

The strong decreasing of $\sigma_{F E}$ for the ${ }^{92} \mathrm{Pd}$, lying close to the p-drip line in the nuclide chart, could also be explained by the opening of new deexcitation channels, such as the multifragmentation one: moving toward the p-drip line a limit may appear in the excitation energy which can be supported by a nucleus, either in the total energy [23] or in the energy per nucleon $[24,25]$. The latter can be related to a limiting temperature for nuclei as derived from

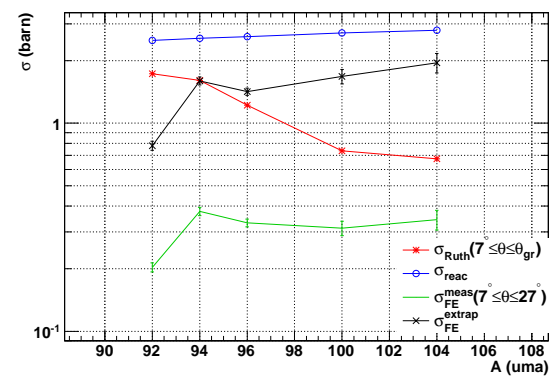

Fig. 5. Integrated Rutherford cross section $\left(\sigma_{\text {Ruth }}\left(7^{\circ} \leq \theta \leq \theta_{g r}\right)\right)$, reaction cross section $\left(\sigma_{\text {reac }}\right)$ and the extrapolated $\left(\sigma_{\text {extrap }}\right)$ cross section as function of the $\mathrm{CN}$ mass is plotted.

Hartree-Fock calculations [26]. In this case one deals with a limit imposed by the exit channel looked at, namely fusion evaporation. This idea is supported by the detection of heavy particles, with $Z$ up to 7 , in coincidence with a residue in VAMOS.

\section{References}

1. P. Donati et al., Phys. Rev. Lett. 72, (1994) 2835

2. T. Rauscher et al., Phys. Rev. C 56, (1997) 1613

3. R. J. Charity et al., Phys. Rev. C 67, (2003) 044611

4. S. I. Al-Quraishi et al., Phys. Rev. C 63, (2001) 065803

5. N. Gelli et al., Search for isospin effects on the level density on nuclei involved in the decay of ${ }^{139} \mathrm{Eu}$ in Recent Achievements and Perspectives in Nuclear Physics: proceeding of the $5^{\text {th }}$ Italy-Japan Symposium (2004)

6. G. Nebbia et al., Phys. Lett. B 176, (1986) 20

7. H. Lehr et al., Nucl. Phys. A 415, (1984) 149

8. H. Morgenstern et al., Phys. Lett. B 113, (1982) 463

9. J. Pouthas et al., Nucl. Instr. and Meth. in Phys. Res. A 357, (1995) 418

10. J. Pouthas et al., Nucl. Instr. and Meth. in Phys. Res. A 369, (1996) 222

11. H. Savajols and VAMOS Collaboration, Nucl. Instr. and Meth. in Phys. Res. B 204, (2003) 146

12. P. Marini, Ph.D. thesis, Universitá di Bologna (2009)

13. B. Gatty et al., Nucl. Phys. A 253, (1975) 511

14. S. T. Hoang and E. Khan, Private communication

15. D.T. Khoa et al., Nucl. Phys. A668, (2000)3

16. W. Nörenberg and H. A. Weidenmüller, Lecture Notes in Physics (Springer-Verlag, 1980)

17. H. Gauvin et al., Phys. Lett. B 58, (1975) 163

18. H. H. Gutbrod, IAEA 2, (1973) 309

19. J. P. Coffin et al., Phys. Rev. C 30, (1984) 539

20. A. Fahli et al., Phys. Rev. C 34, (1986) 161

21. B. Sikora et al., Phys. Rev. C 25, (1982) 1446

22. W. Scobel et al., Phys. Rev. C 14, (1976) 1808

23. B. Borderie et al., Z. Phys. A 321, (1985) 703

24. W. Bohne et al., Phys. Rev. C 41, (1990) R5

25. L. Lassen et al., Phys. Rev. C 55, (1997) 1900

26. P. Bonche et al., Nucl. Phys. A 436, (1985) 265 